\title{
Cryptomenorrhea with cervicovaginal aplasia: endoscopic transfundal development of the lower genital tract
}

\author{
Ali M. El Saman • Magdi M. Amin • \\ Mohamad T. Khalaf • Dina M. Habib • \\ Omar M. Shaaban • Alaa M. Ismail
}

Received: 7 November 2013 / Accepted: 26 August 2014 / Published online: 16 September 2014

(C) Springer-Verlag Berlin Heidelberg 2014

\begin{abstract}
The current case series was done to evaluate the feasibility of transfundal hysteroscopy (TFH) in helping the development of new cervical canal in cases with cervical aplasia. Five cases with obstructive cervicovaginal agenesis with hematometra were included in this report. Laparoscopicguided TFH was done in conjunction with endoscopic canalization to all cases. Additional retropubic balloon vaginoplasty (BV) was needed in three cases with associated vaginal aplasia. The hysteroscope was passed through the uterine fundus. After complete washing, the endometrial lining was inspected, and a properly located intrauterine catheter coming out from the vagina or vaginal dimple was used to drain the uterine cavity and maintain the cervical tract. The procedure was done successfully in all cases with adequate drainage of hematometria. Additional time needed for TFH was between 4 to $15 \mathrm{~min}$. Second-look hysteroscopy revealed adequate canalization in all cases. The five cases had regular menstrual cycles up to the sixth postoperative month. In conclusion, TFH is a safe and feasible procedure as a harmonizing technique during endoscopic canalization of cervical atresia with or without BV in cases of obstructive Müllerian anomalies. The procedure accelerates the drainage of uterine contents and localizes the correct site of draining catheter.
\end{abstract}

Condensation Laparoscopic transfundal hysteroscopy was performed for patients with cervical/cervicovaginal aplasia to examine uterine cavity, facilitate washing of uterine contents, and decide the proper location of the draining catheter

A. M. El Saman • M. T. Khalaf • D. M. Habib •

O. M. Shaaban $(\triangle) \cdot A$. M. Ismail

Department of Obstetrics and Gynecology, Faculty of Medicine,

Assiut University, P.O. Box 174, Assiut, Egypt

e-mail: omshaaban2000@yahoo.com

M. M. Amin

Department of Obstetrics and Gynecology, Faculty of Medicine,

Sohag University, Sohag, Egypt
Keywords Balloon vaginoplasty $\cdot$ Neovagina $\cdot$ Laparoscopy $\cdot$ Vaginal aplasia $\cdot$ Cervical aplasia $\cdot$ Hysteroscopy $\cdot$ Hematometra $\cdot$ Transfundal hysteroscopy

\section{Introduction}

Müllerian aplasia represents a wide spectrum of disorders, which are occasionally associated with functioning uterus and menstrual retention. Balloon vaginoplasty (BV) was introduced by El-Saman and coworkers as scar-free procedure for construction of a new sexual port that mimics the normal vagina in several ways [1-3]. The patients with isolated vaginal or cervicovaginal aplasia and functioning uteri are often treated by laparotomies or advanced laparoscopic operations. In our previous reports, such patients were successfully treated by $\mathrm{BV}$ in conjunction with endoscopically monitored introduction and fixation of a silicon catheter through the vaginal dimple. The catheter drains the retained bloody contents of the uterus for 6-month period required for complete establishment of a communicating tract [4-6]. In these cases, the proper placement of the intrauterine catheter/drain is the key of success.

The present report represents the first trial to use the conventional rigid hysteroscopy in a transfundal visualization of the uterine cavity and localization of the proper site of catheter or the silicon drain insertion in women with vaginal or cervicovaginal aplasia with functioning uteri.

\section{Patients and methods}

This is a case series performed in the Women's Health Hospital of the Assiut Medical School during the period from May 2012 to May 2013. The study had obtained the ethical clearance of the Institutional Ethical Review Board. The study 
comprised five adolescent patients. They all had complete vaginal and/or cervical aplasia; two of them were classed as UOC4V0 and three as U0C4V4 according to ESHRE/ESGE classification system [7]. All patients presented with cyclic pains secondary to menstrual retention. Complete history was taken and a vigilant examination with objective measurements of the depth of the vaginal dimple. We counseled our participants for having the balloon vaginoplasty and endoscopic canalization (BV-EC) with inspection of the uterine cavity via transfundal hysteroscopy (TFH). This is in a trial of creation of new cervical or cervicovaginal tract to correct their congenitally malformed lower genital tract and to establish their reproductive functions.

A written consent was obtained from each participant after reading the patient's information sheet that includes detailed explanation of the procedure including benefits and possible risks. Laparoscopically guided TFH was done in conjunction with canalization to all cases in this series. The procedure was carried out under general anesthesia with endotracheal tube. The patients were sterilized and draped in a dorsal lithotomy position and thoroughly examined. Preoperative antibiotic was given in all cases. The procedure started by laparoscopy in all cases using a 5-mm intra-umbilical port. Detailed examination of the abdominal and pelvic organs was performed to confirm the diagnosis and the exact localization of the site of aplasia and estimate the missing aplastic portion of the genital tract.

A specially designed reusable sterilized inserter, which was designed to perforate the vaginal dimple in cases of BV in women with Müllerian agenesis, was used to make a uterine fundal hole. The inserter is $35 \mathrm{~cm}$ long and $4 \mathrm{~mm}$ girth and is made of stainless steel. It has a sharp penetrating pyramidal tip with a long double strand silk suture (DSSS) threaded into its fenestrated caudal end. After penetrating the uterine fundus with the inserter under laparoscopic guidance, the inserter passes across the distended uterine cavity to pass through the atretic segment of the cervix to be finally extracted through the vagina in cases with isolated cervical atresia or through vaginal dimple in cases with concomitant vaginal aplasia. A silicon catheter is then inserted from the vaginal side through the hole made by the inserter and manipulated into the uterine cavity as described in our original procedure. The catheter was pulled up into the uterine cavity via the DSSS. The inserter and the technique used in BV-EC was previously described [5].

Then, under laparoscopic guidance, a 4-mm rigid hysteroscopy was passed across an ancillary midline port then through the same puncture of the inserter across the uterine fundus to inspect the uterine cavity as shown in Fig. 1. Continuous irrigation of the uterine cavity was made using saline solution pushed through the TFH until clear fluid came out through the intrauterine catheter coming out from the vaginal side (Fig. 2). Detailed hysteroscopic inspection of the uterine cavity was done including visualization of the tubal ostia when $70^{\circ}$ hysteroscopic lens was used. The position of the balloon was ascertained to confirm its accurate intrauterine placement. After confirmation of the intrauterine location of the catheter, the hysteroscopy was withdrawn. Laparoscopic inspection of the access site at the uterine fundus was done for detection of and management of any bleeding from the edges.

The three cases with associated vaginal aplasia (U0C4V4) required additional retropubic balloon vaginoplasty (previously described in our early series) [8] to increase the length of the vaginal dimple and carry it up to meet the newly formed cervix.

The above-described inserter was passed again just behind the symphysis pubis carrying a catheter on its caudal end. The catheter was passed across the retropubic space until the balloon-bearing segment appears at the vaginal dimple (Fig. 3a). The balloon was distended with $20 \mathrm{~mm}$ saline and traction was initiated until disappearance of the balloon within the vaginal introitus. Support of the catheter in place after traction was done using a supporting plate placed over a dressing on the abdominal wall retained in its place by sterile umbilical cord clamp (Fig. 3b). This is followed by $1-2 \mathrm{~cm}$ daily increase in the traction exerted on the catheter by adding another proximal clamp. Pulling up on the retropubic balloon was coupled with pulling down on the uterine catheter until the two balloons become in contact, a process that was monitored via transrectal ultrasound (Fig. 4). More details about retropubic BV can be found in a previous publication [8]. When vaginal depth increased to $9-10 \mathrm{~cm}$ on days $6-7$, the retropubic catheter was removed and uterine catheter was exchanged with new silicon one. The uterine catheter was changed every 30 to 40 days during that change; office hysteroscopy was done to ensure the patency of the cervical track. The catheter was finally removed after 4 to 6 months of the procedure and complete epithelization of the cervical tract had been visualized through the hysteroscopy.

\section{Results}

The mean age of the five patients was $13.1 \pm 1.1$ years. Their main complaint was recurrent cyclic lower abdominal pains and amenorrhea. None of them was married at the time of operation. One of them had two previous attempts of uterovestibular anastomosis and had severe abdominal pain and fever with lower abdominal tenderness. Details of patients' clinical and operative data are summarized in Table 1. The canalization procedure was performed successfully in the five cases. Endoscopic transfundal canalization was done alone in cases with isolated cervical aplasia with rather adequate vaginal length. However, the canalization was done in conjunction with retropubic BV in three cases with cervicovaginal aplasia. 

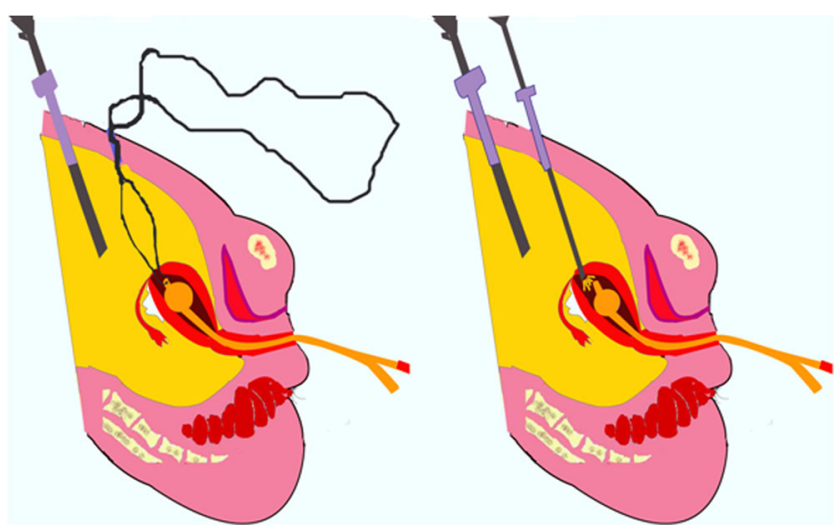

Fig. 1 A long metal inserter with a long double strand of silk suture is being inserted transfundal under laparoscopic monitoring

Transfundal hysteroscopy was successfully performed for the five cases. The operative time of TFH ranged between 5 and $14 \mathrm{~min}$. Most of the time was spent in drainage and washing out the uterine contents. The uterine contents were thick chocolate blood in two cases, liquid dark blood in two cases, and creamy colored infected contents in one case. The endometrium was healthy in four cases and covered with pyogenic membrane in the infected case. The irrigation was continued until most of the pyogenic membrane was detached and washed out. The intrauterine location of balloon was confirmed in all cases. Tubal ostia were visualized in three cases (when the $70^{\circ}$ telescope was available); in the other two cases, $30^{\circ}$ telescope was used which not allowing visualization of the ostia. Inspection of the access site at the fundus was done for all cases and confirmed the absence of any significant

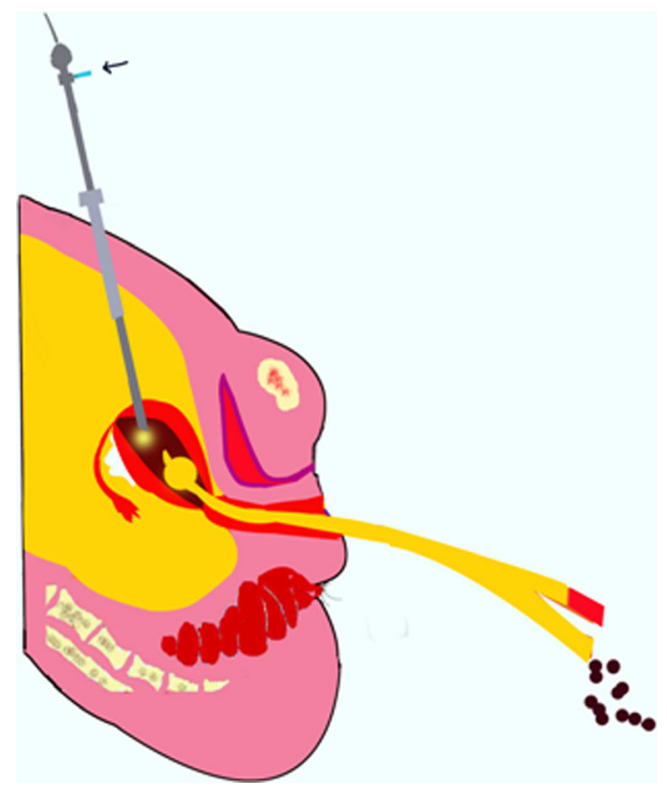

Fig. 2 Transfundal hysteroscopy is being passed through the already present fundal puncture; intrauterine contents going to be washed under vision. A probably placed intrauterine catheter is draining the uterine cavity and establishing the cervical canal

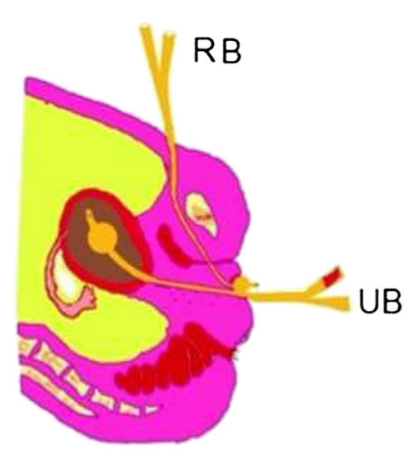

A

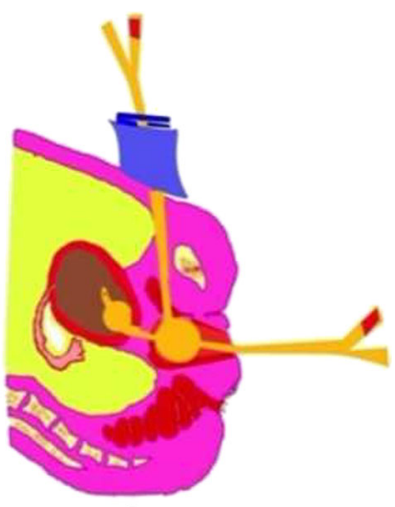

B
Fig. 3 Combined TFH canalizations with intrauterine catheter draining the uterine cavity coming out from the vaginal dimple, and retropubic vaginoplasty with the catheter coming out from the anterior abdominal wall in cases with cervicovaginal aplasia. a Catheters before traction. b Catheters after traction. $U B$ uterine balloon, $R B$ retropubic balloon

bleeding. None of the cases required suturing of the fundal puncture. Bladder or rectal injuries have not occurred during any of the procedures.

In the cases with vaginal aplasia (U0C4V4), combined upward and downward traction was monitored via transrectal ultrasound until the uterine and retropubic balloons became in contact; kissing balloons was achieved (Fig. 4).

Day 7 second-look transvaginal office hysteroscopy was done across the new vagina and via the catheter tract during catheter change in three cases. The puncture site at the fundus was invisible and the whole fundus was lined by healthy endometrium. Uterine silicone catheters were changed three to five times and were retained for 3-6 months. Hysteroscopic examination of the communication tract and uterine cavity revealed epithelization of the tract from the first postoperative menstrual period. One case got married 6 months after the procedure, her follow-up data as regard the sexual penetration and satisfaction scores were give in Table 1.

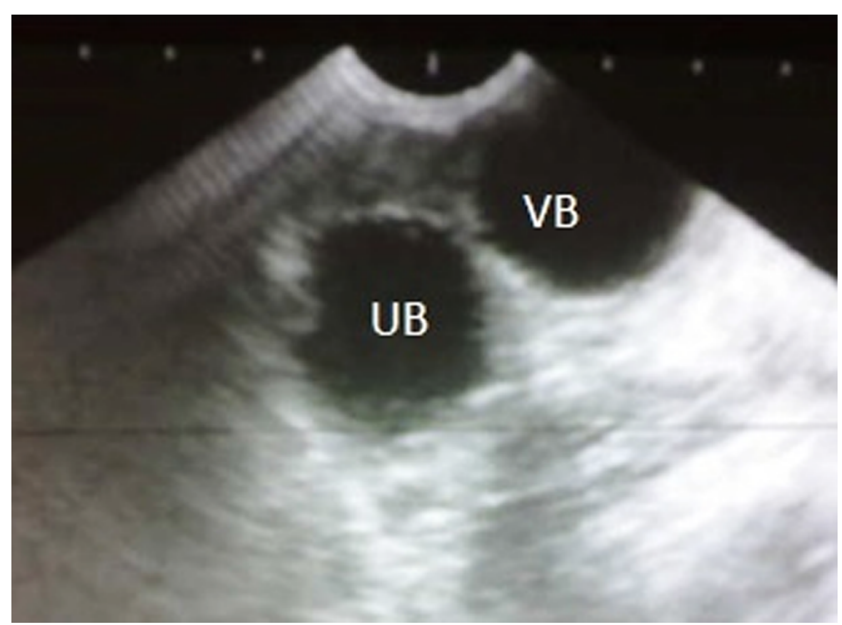

Fig. 4 Transrectal ultrasounds scan showing the uterine balloon $(U B)$ in close contact with retropubic balloon $(V B)$ 
Table 1 Clinical findings, type of the procedure, and outcomes in the five cases undergone transfundal hysteroscopy

\begin{tabular}{|c|c|c|c|}
\hline Findings & Infected hematometra (1 case) & Thick chocolate blood ( 2 cases) & Thin chocolate blood ( 2 cases) \\
\hline Presentation & Fever, pains, and tenderness & Cyclic pains & Cyclic pains \\
\hline Previous surgeries & $\begin{array}{l}2 \text { conventional trials of utero-vestibular } \\
\text { anastomosis }\end{array}$ & Appendectomy & $\begin{array}{l}\text { Appendectomy } 1 \text { case } \\
\text { Laparoscopy in the other }\end{array}$ \\
\hline Type of anomaly & Cervicovaginal aplasia (U0C4V4) & $\begin{array}{l}\text { Cervicovaginal aplasia } \\
\text { (U0C4V4) }\end{array}$ & $\begin{array}{l}\text { Isolated cervical aplasia } \\
\quad(\mathrm{U} 0 \mathrm{C} 4 \mathrm{~V} 0)\end{array}$ \\
\hline Type of procedure & Canalization + RBV & Canalization + RBV & Canalization only \\
\hline \multicolumn{4}{|l|}{ Operative time (min) } \\
\hline Total & 70 & 55 and 65 & 40 and 35 \\
\hline TFH & 14 & 13 and 12 & 5 and 6 \\
\hline Endometrium & Covered with pyogenic membrane & Healthy & Healthy \\
\hline Menstrual flow during follow-up & $\begin{array}{l}6 \text { months } \\
\text { Regular menstruation }\end{array}$ & $\begin{array}{l}18 \text { and } 25 \text { months } \\
\text { Regular menstruations }\end{array}$ & $\begin{array}{l}22 \text { and } 28 \text { months } \\
\text { Regular menstruations }\end{array}$ \\
\hline Cyclic pains & Dramatic relive & Dysmenorrhea & Relived \\
\hline Neovaginal depth (cm.) & 9 & 9 and 10 & - \\
\hline \multicolumn{4}{|l|}{ Penetration scores (..$/ 100$ points $)$} \\
\hline $\begin{array}{l}\text { Husband } \\
\text { Wife }\end{array}$ & - & $\begin{array}{l}80 \\
80\end{array}$ & - \\
\hline \multicolumn{4}{|l|}{ Satisfaction scores (.../100 points) } \\
\hline $\begin{array}{l}\text { Husband } \\
\text { Wife }\end{array}$ & - & $\begin{array}{l}90 \\
90\end{array}$ & - \\
\hline
\end{tabular}

$T F H$ transfundal hysteroscopy, $R B V$ retropubic balloon vaginoplasty

\section{Discussion}

In the present work, we describe the use of rigid hysteroscopy through a transfundal approach during the endoscopic managements of cervical and or cervicovaginal atresia. The procedure was performed alone in women with isolated cervical atresia and in conjunction with retropubic BV in cases associated with vaginal aplasia. The procedure was done successfully in the five cases reported in this case series. The operations were followed by resumption of regular menstruation, relive of pain in all operated cases, and by normal sexual relations in one of the cases who got married.

Obstructive Müllerian anomalies (OMAs) represent a challenging category of Müllerian duct anomalies [7, 8]. In this case series, OMAs cases secondary to isolated cervical atresia/ aplasia were treated with a simple endoscopic canalization technique without laparotomy or any dissection at the lower pole of the uterus or at the vaginal apex.

Compared to open surgical procedures [9-11] that were either performed via exploratory laparotomy or assisted by laparoscopy, the present procedure does not entail any dissection. Absence of dissection may be an important attributing factor to the long-lasting success that was achieved $[5,6]$. Cases with OMAs associated with vaginal aplasia (U0C4V4) on the other hand were treated with endoscopic canalization using TFH in conjunction with retropubic BV [6]. In both circumstances, placement of intra-cervical stent was crucial for success. Silicone catheters gauge 24-25 have a good caliber and less compressible stem that was used as a selfretaining cervical stent after inflation of its intrauterine placed balloon. Precise balloon placement inside the uterine cavity is an important key point for successful outcome.

In the present report, confirmation of accurate balloon placement was done via transfundal hysteroscopic examination. The visualization of whole circumference of balloon inside the uterine cavity was considered as an adequate evidence of its correct intrauterine location. Furthermore, the introduction of hysteroscopy with its pressurized fluid distention media allowed expedite and complete drainage of all thick retained menstrual blood that might minimize the chances of subsequent infection. In the single patient with concurrent infection, hysteroscopy distention media allowed not only rapid and complete drainage of pus and infected blood but also allowed detachment and washout of the pyogenic membrane. Dramatic relief of fever and improvement of the associated pain followed the procedure.

At the first glance, TFH can be considered as an invasive procedure since it was carried out through an intentional fundal perforation. However, the procedure itself did not add any invasive steps as the puncture of the myometrium was already created during the process of canalization and catheter placement. Therefore, the intended uterine puncture was a fundamental step of the original canalization procedure and the hysteroscopy was just passed through the preformed passage. 
There are several other described successful procedures for management of cervicovaginal aplasia [9-13]. However, dissection either abdominal or vaginal is a fundamental part of these procedures. Therefore, subsequent scar formation is an anticipated consequence. If contracture of the scar develops, long-term failure of the procedure might result. In addition, subsequent surgeries will be more difficult. On contrast, our scalpel-free endoscopic procedures are not associated with any dissection and expected to have an expanding role for future management of OMAs.

The weakness point of the present report is the limited number of cases. However, there are some favorable points beside the aforementioned immediate benefits. This endoscopic development of lower genital tract opens new perspective for lesser invasive surrogate access to the uterine cavity whenever the natural access is impossible.

Conflict of interest Ali El Saman, Magdi Amin, Mohamad Khalaf, Dina Habib Omar Shaaban, and Alaa Ismail declare that they have no conflict of interest.

Informed consent All procedures followed were in accordance with the ethical standards of the responsible committee on human experimentation (institutional and national) and with the Helsinki Declaration of 1975 , as revised in 2000. Informed consent was obtained from all patients for being included in the study.

\section{References}

1. El Saman AM (2010) Retropubic balloon vaginoplasty for management of Mayer-Rokitansky-Kuster-Hauser syndrome. Fertil Steril 93: 2016-2019

2. El Saman AM, Fathalla MM, Nasr AM, Youssef MA (2007) Laparoscopically assisted balloon vaginoplasty for management of vaginal aplasia. Int J Gynaecol Obstet 98:134-137
3. El Saman AM, Fathalla MM, Zakherah MS, Shaaban OM, Nasr A (2009) Modified balloon vaginoplasty: the fastest way to create a natural: minor changes in technique eliminate the need for customized instruments. Am J Obstet Gynecol 201(546):e1e5

4. El Saman AM, Habib DM, Othman EE, Tawfik RM (2011) Successful canalization of a noncommunicating uterine horn by horn-vaginal anastomosis: preliminary findings of a novel approach for an unclassified anomaly. J Pediatr Surg 46:14641468

5. El Saman AM (2010) Endoscopically monitored canalization for treatment of congenital cervical atresia: the least invasive approach. Fertil Steril 94:313-316

6. El Saman AM (2009) Combined retropubic balloon vaginoplasty and laparoscopic canalization: a novel blend of techniques provides a minimally invasive treatment for cervicovaginal aplasia. Am J Obstet Gynecol 201(333):e1-e5

7. Grimbizis GF, Gordts S, Di Spiezio Sardo A, Brucker S, De Angelis C, Gergolet M, Li TC, Tanos V, Brolmann H, Gianaroli L, Campo R (2013) The ESHRE/ESGE consensus on the classification of female genital tract congenital anomalies. Hum Reprod 28:2032-2044

8. El Saman AM, Nasr A, Tawfik RM, Saadeldeen HS (2011) Mullerian duct anomalies: successful endoscopic management of a hybrid bicornuate/septate variety. J Pediatr Adolesc Gynecol 24:e89-e92

9. Roberts CP, Rock JA (2011) Surgical methods in the treatment of congenital anomalies of the uterine cervix. Curr Opin Obstet Gynecol 23:251-257

10. Rock JA, Roberts CP, Jones HW Jr (2010) Congenital anomalies of the uterine cervix: lessons from 30 cases managed clinically by a common protocol. Fertil Steril 94:1858-1863

11. Grimbizis GF, Tsalikis T, Mikos T, Papadopoulos N, Tarlatzis BC, Bontis JN (2004) Successful end-to-end cervico-cervical anastomosis in a patient with congenital cervical fragmentation: case report. Hum Reprod 19:1204-1210

12. Deffarges JV, Haddad B, Musset R, Paniel BJ (2001) Utero-vaginal anastomosis in women with uterine cervix atresia: long-term followup and reproductive performance. A study of 18 cases. Hum Reprod 16:1722-1725

13. Fliegner JR, Pepperell RJ (1994) Management of vaginal agenesis with a functioning uterus. Is hysterectomy advisable? Aust N Z J Obstet Gynaecol 34:467-470 